\title{
TEMA 2-2016: Amenaza de Parto Pretérmino
}

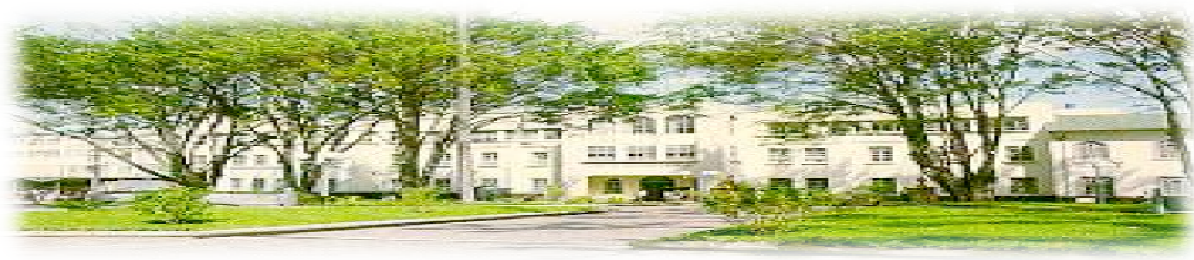

Hospital San Juan de Dios, San José, Costa Rica. Fundado en 1845

Recibido:

Aceptado:
$20 / 11 / 2015$

$06 / 12 / 2015$

Gabriela Quirós González ${ }^{1}$

Raúl Alfaro Piedra ${ }^{2}$

Mariela Bolívar Porras ${ }^{3}$

Natalia Solano Tenorio ${ }^{4}$

${ }^{1}$ Médico Cirujano, Área de Salud Guatuso, Caja Costarricense de Seguro Social. Correo: gabrielaqg@gmail.com

${ }^{2}$ Medico Asistente en Ginecología y Obstetricia, Hospital Calderón Guardia, Caja Costarricense de Seguro Social

${ }^{3}$ Médico Cirujano, Área de Salud Guápiles, Caja Costarricense de Seguro Social

${ }^{4}$ Médico Cirujano, Área de Salud Belén - Flores, Caja Costarricense de Seguro Social

\section{RESUMEN}

La amenaza de parto pretérmino, consiste en la presencia de contracciones uterinas regulares asociadas a modificaciones cervicales, que ocurren después de las 20 y antes de las 37 semanas de gestación, independientemente del peso al nacer, y es de causa multifactorial. A pesar de que se ha logrado disminuir su frecuencia, debido a las mejoras existentes en prácticas de fertilidad y el aumento de estrategias que buscan la prevención de esta patología; sigue siendo una de las principales causas de mortalidad y morbilidad infantil, lo que ocasiona secuelas a corto y largo plazo, y lo convierte en una importante problema de salud pública. Debido a esto es trascendental reconocer a todas aquellas mujeres que tengan riesgo de presentar una amenaza de parto pretérmino, principalmente aquellas que ya tu- vieron antecedente de un parto prematuro. Sin embargo en muchas ocasiones no es posible encontrar factores de riesgo por lo que se debe tener conocimiento de las diferentes herramientas clínicas, diagnósticas y terapéuticas de esta patología para poder así realizar un diagnostico rápido y tomar las medidas necesarios del caso, ya que la sobrevida neonatal aumenta progresivamente con la edad gestacional, por lo que cada día tiene un impacto crítico en esta, además disminuye el riesgo de mortalidad y de complicaciones. El siguiente manuscrito consiste en una revisión bibliográfica de la amenaza de parto pretérmino, en cuanto a sus generalidades, factores de riesgo, etiología, métodos diagnósticos y tratamiento.

PALABRAS CLAVE 
parto prematuro, amenaza de parto pretérmino, fibronectina, longitud cervical

\section{ABSTRACT}

Preterm labor is the treat of early labor, that is defined by the presence of regular uterine contractions associated with cervical changes that occur after 20 weeks and before 37 weeks of gestation, regardless of the weight at birth, and associated with a multifactorial cause. Although the frequency of this disease has been reduced, mainly due to the improvements in existing fertility practices and increased strategies that seek to prevent it; Pretem Labor remains one of the leading causes of morbidity and infant mortality, causing short and long term sequels, and its considered an important public health problem. Because of this, it is important to recognize those women who have a risk of having a preterm labor, especially those with a history of premature labor. Nevertheless, it is not always possible to find the presence of risk factors, it is important to be aware of the various clinical, diagnostic and therapeutic tools available and to make a quick diagnosis to be able to take necessary measures if presented with such a case, because neonatal survival increases progressively with gestational age, every day has a critical impact and reduces the risk of death and complications. The following manuscript consists of a review in texts pertaining to preterm labor, in its general aspects, risk factors, etiology, diagnosis and treatment methods.

\section{KEY WORDS}

preterm birth, preterm labor, fibronectin, cervical length

\section{INTRODUCCIÓN}

\section{Generalidades}

Un nacimiento prematuro se define comúnmente como aquel que ocurre después de las 20 semanas de gestación y antes de finalizar las $37 \mathrm{se}-$ manas, independientemente del peso al nacer. ${ }^{(1,2)}$ De la misma manera, Amezada de Parto Pretérmino (APP) consiste en la presencia de contracciones uterinas regulares en este mismo rango de tiempo, asociado a cambios progresivos del cérvix, tales como dilatación y borramiento característicos. ${ }^{(3,4)}$

La prematuridad constituye una de las principales causas de morbimortalidad neonatal y es responsable de un gran porcentaje de secuelas infantiles. ${ }^{(5)}$ Constituye aproximadamente el $70 \%$ de la mortalidad perinatal y el $75 \%$ de la morbilidad. ${ }^{(6)}$ La Organización Mundial de la Salud (OMS) estimó que el 9,6\% de todos los nacimientos en 2005 fueron prematuros. ${ }^{(1)}$

Sin embargo, se debe recalcar que se ha producido un descenso de la tasa de nacimientos prematuros; ya que se disminuyó a $12.7 \%$ en 2007 , $12,3 \%$ en 2008 y $12,1 \%$ en 2009 . Esta disminución se explica por la mejora de las prácticas de fertilidad que reducen el riesgo de gestaciones múltiples y el aumento en el uso de estrategias para prevenir el parto prematuro recurrente. ${ }^{(1)}$

Un dato que se debe recordar es que los afroamericanos tienen tasas de nacimientos prematuros casi dos veces mayores que otros grupos étnicos. ${ }^{(1)}$

Es importante tener en cuenta que la sintomatología de la amenaza de parto pretérmino (APP) es muy imprecisa y muchos de estos síntomas han sido asignados de manera empírica a parto prematuro inminente. ${ }^{(5,7)}$

El parto pretérmino es un problema para la salud pública a nivel mundial, ya que conlleva complicaciones neonatales a corto plazo, como depresión al nacer, síndrome de dificultad respiratoria, hemorragia intraventricular, sepsis, trastornos metabólicos, enterocolitis necrotizante, ductus arterioso persistente, displasia broncopulmonar o apneas. A largo plazo se dan parálisis cerebral, retraso mental, compromiso de visión y pérdida de audición. ${ }^{(8)}$

Por consiguiente, la sobrevida neonatal es dependiente de la madurez del neonato y aumenta progresivamente con la edad gestacional, por lo que cada día impacta críticamente y disminuye el riesgo de mortalidad y complicaciones. ${ }^{(9)}$

\section{Factores de riesgo}

Lograr la identificación de factores de riesgo para nacimientos pretérmino, antes de la concepción o tempranamente en el embarazo, conduciría a realizar intervenciones que podrían ayudar a prevenir un parto pretérmino. No obstante, realizar esta identificación temprana es difícil debido a que un número importante de nacimientos pretérminos ocurre en mujeres que no tienen factores de riesgo. ${ }^{(10)}$ 
Además, algunas complicaciones obstétricas que terminan en parto pretérmino requieren de cofactores para ejercer sus efectos, haciendo que la cadena de causalidad sea difícil de documentar. Finalmente, aún no existe un adecuado modelo animal para lograr estudiar el parto pretérmino. ${ }^{(9)}$ Es importante tener en cuenta que el factor de riesgo más importante para predecir un parto pretérmino es tener el antecedente de parto pretérmino ${ }^{(11)}$, el cual representa un $15 \%$ después de uno y $30 \%$ si fueron dos partos pretérmino. Este antecedente incrementa de 1,5 a 2 veces el riesgo. ${ }^{(12)}$

Algunos de los factores de riesgo identificados son: ${ }^{(1,9,12,13,14)}$

-Antecedentes de pérdidas gestacionales en el segundo trimestre, aborto habitual, anomalías uterinas, conización del cérvix, longitud cervical corta.

-En el anteparto: embarazos múltiples, ruptura prematura de membranas, polihidramnios, hemorragia anteparto, cirugía intraabdominal, infección del tracto urinario, infección materna severa, trauma físico emocional.

-Factores y condiciones maternas: infección bacteriana ascendente, enfermedades maternas como hipertensión arterial, diabetes mellitus o gestacional, trombofilias, entre otras, infecciones transplacentarias como sífilis, enfermedad periodontal, edad materna $\leq 18 \mathrm{a} \geq 40$ años.

-Factores epidemiológicos y ambientales, factores socioeconómicos (bajo nivel socioeconómico) y psicosociales, etnia afroamericana $(18,4 \%)$, tabaquismo, factores nutricionales como bajo peso materno, índice de masa corporal menor de 19,8 y periodo intergenésico corto.

\section{Etiología}

Se conoce que la amenaza de parto pretérmino puede tener un origen multifactorial. Sin embargo, tradicionalmente sus posibles causas se han seleccionado en tres grandes grupos: ${ }^{(15)}$

-Iatrogénico: por indicación médica se finaliza el embarazo antes del término. Ejemplo: secundario a alguna patología materna, que impida lograr el avance de la gestación.

-Secundario a Rotura Prematura de Membranas (RPM)

-Idiopático: sin causa aparente o conocida.

Recientemente, según experiencias clínicas y experimentales, la mayoría de los partos pre- términos secundarios a causas idiopáticas y por RPM se ligan a cuatro procesos: ${ }^{(9)}$

a) Activación del eje hipotálamo-pituitariaadrenal materno o fetal.

b) Inflamación decidual y amniocoriónica.

c) Hemorragia decidual.

d) Distensión uterina patológica: embarazos múltiples y polihidramnios.

Los procesos mencionados generalmente ocurren simultáneamente; sin embargo, cada uno tiene una característica única bioquímica.

Estos procesos convergen en una vía final común en donde se produce liberación de mediadores bioquímicos, aumento de proteasas y uterotoninas de membranas fetales y decidua. Por consiguiente, se produce el inicio de las contracciones uterinas con modificaciones del cérvix, con o sin RPM, y finalmente se desencadena el parto prematuro. ${ }^{(16)}$

\section{Diagnóstico y evaluación clínica}

Para realizar el diagnóstico de la APP es preciso constatar la aparición de los siguientes parámetros, entre las semanas $20^{\mathrm{a}}$ y $37^{\mathrm{a}}$ de gestación, e independientemente de la integridad o no de las membranas: ${ }^{(17)}$

-Contracciones uterinas: debe tenerse en cuenta la posibilidad de parto pretérmino en todas las mujeres que presenten síntomas de hiperactividad uterina, al menos cuatro contracciones en 20 minutos, $u$ ocho en 60 minutos, antes de la semana $37^{\text {a(17) }}$, las cuales se pueden manifestar como contracciones dolorosas o indoloras, presión pélvica, dolor lumbar, sangrado vaginal, entre otras. ${ }^{(1)}$ Sin embargo, los signos y síntomas son poco específicos, ya que es normal que las gestantes presenten contracciones durante su embarazo y estas, al ser indoloras, pueden confundirse con las de Braxton Hicks, diferenciándose únicamente por su persistencia. ${ }^{(17)}$

-Modificación cervical: se ha asociado con el parto prematuro la existencia de una dilatación cervical $>2 \mathrm{~cm}$ y/o un borramiento $>80 \%{ }^{(17)} \mathrm{La}$ exploración clínica del cérvix es subjetiva e inexacta, por lo cual tiene una reproducibilidad limitada entre los examinadores. Por este motivo se recomienda no utilizar el examen clínico del cérvix de manera rutinaria para evaluar pacientes con riesgo de amenaza de parto prétermino. ${ }^{(1)}$ 
-Pruebas diagnósticas

Ecografía transvaginal y Longitud cervical: Se realizará entre la semana 20 y 34 de gestación $^{(1)}$, si es posible como complemento del examen cervical, si la contractibilidad no es franca o las modificaciones cervicales son dudo$\mathrm{sas}^{(8)}$. Una longitud cervical $<25 \mathrm{~mm}$ se asocia con un riesgo relativo de parto prematuro de 4,8 . El mayor valor predictivo positivo se obtiene con una longitud cervical inferior a $18 \mathrm{~mm}$ y el mejor valor predictivo negativo con una longitud superior a $30 \mathrm{~mm}$. Por lo tanto, una longitud cervical de $25 \mathrm{~mm}$ en presencia de contracciones con las características mencionadas anteriormente es diagnóstico de APP. Si la longitud cervical es mayor, la probabilidad de APP es muy baja independientemente de la frecuencia de contracciones. ${ }^{(17)}$

Test de fibronectina: Se realizará entre las semanas 24-34 de edad gestacional en pacientes con riesgo de parto pretérmino. ${ }^{(11)} \mathrm{La}$ fibronectina es una proteína extracelular que se encuentra en las membranas, decidua y líquido amniótico, con una función adhesiva entre el feto en desarrollo y la superficie interna del útero (interfase coriodecidual). Tras la implantación del saco gestacional aparece fibronectina en las secreciones cervicovaginales. Puede encontrarse normalmente en ellas antes de la semana $20^{\mathrm{a}}-22^{\mathrm{a}}$ y al final del embarazo. Su presencia entre las semanas $24^{\mathrm{a}}$ y $34^{\mathrm{a}}$ es poco frecuente, y puede indicar separación de las membranas fetales de la decidua. ${ }^{(17)} \mathrm{Un}$ valor mayor a $50 \mathrm{ng} / \mathrm{ml}$ representa un riesgo incrementado de amenaza de parto pretérmino. ${ }^{(8)}$

Ambas pruebas tienen un alto valor predictivo negativo, de ahí su importancia, por lo tanto el uso único o de ambas permite determinar cuáles pacientes necesitarán ser hospitalizadas y recibir tratamiento tocolítico. ${ }^{(11)}$

\section{Tratamiento}

-Medidas generales: se ha demostrado que el reposo en cama y la hidratación no disminuyen la frecuencia de nacimientos pretérmino, por lo cual no deben recomendarse rutinariamente. ${ }^{(9)}$

-Progesterona: debe ser usada para la prevención del parto pretérmino en pacientes con antecedente de dicha entidad. Su función es mantener la quiescencia uterina mediante la supresión de los efectos proinflamatorios de los estrógenos. Es preferible utilizarlo vía vaginal, iniciando entre la 16-24 semana de gestación. Su uso ha demostrado disminución en las tasas de parto pretérmino de $45 \%$ en pacientes con cuello corto.

-Tocolíticos: El uso de tocolíticos no se asocia con la reducción de la mortalidad perinatal y neonatal o de la morbilidad neonatal ${ }^{(12)}$.Su principal indicación es para las mujeres que están en amenaza de parto pretérmino, y de esta forma prolongar el nacimiento, por lo menos en 48 horas, para permitir el efecto óptimo de los glucocorticoides antenatales que disminuyen la morbilidad y mortalidad de los recién nacidos prematuros. No está definido un tocolítico de primera elección para el manejo de parto pretérmino. ${ }^{(13)}$ Las circunstancias clínicas, la preferencia y experiencia del médico dictarán el tratamiento. ${ }^{(9)}$

Los tocolíticos disponibles para el manejo de la amenaza de parto pretérmino son: calcioantagonistas, antiinflamatorios no esteroideos (AINES), agonistas de los receptores beta adrenérgicos (terbutalina), sulfato de magnesio, e inhibidores de los receptores de oxitocina (atosiban). El Colegio Americano de Ginecología y Obstetricia recomienda como tratamiento de elección los calcioantagonistas; el nifedipino es el más utilizado por tener menos efectos colaterales, los AINES y los agonistas B adrenérgicos. ${ }^{(12)}$ Sin embargo, el atosiban y la indometacina, que deben usarse antes de las 32 semanas y no más de 48 horas, son los que presentan menos efectos adversos, por lo cual son en muchas ocasiones el tratamiento de primera línea ${ }^{(9)}$. Dentro de las contraindicaciones de tocolisis se encuentran: preeclampsia y eclampsia, malformaciones congénitas o cromosómicas letales, abruptio placenta, dilatación cervical avanzada, insuficiencia placentaria y sangrado materno con inestabilidad hemodinámica. ${ }^{(1,12)}$

Corticoides: según revisiones de Cochrane, los corticoides reducen la morbilidad y mortalidad neonatal en $34 \%$ y $31 \%$, respectivamente ${ }^{(12)}$, ya que estimulan el desarrollo pulmonar por medio de la síntesis de factor surfactante, aumentan la compliance pulmonar y disminuyen la permeabilidad vascular. ${ }^{(1)}$ Son candidatas a corticoides toda mujer embarazada con alto riesgo de parto pretérmino entre las 24 y 34 semanas de gestación.

Se pueden incluso indicar corticoesteroides sobre las 34 semanas si existe evidencia de inmadurez pulmonar fetal. Toda mujer que curse con las condiciones mencionadas anteriormente debe ser 
tratada siempre que el parto no sea inminente (menos de una hora). Pueden tratarse mujeres embarazadas con ruptura prematura de membranas siempre que no existan signos de corioamnionitis, tampoco es contraindicación la diabetes gestacional pero requiere estricta monitorización de la glicemia. La terapia recomendada es betametasona $12 \mathrm{mg}$ IM separadas por 24 horas. Otra terapia alternativa es la dexametasona, $6 \mathrm{mg}$ cada 12 horas cuatro dosis. Según la Academia Americana de Ginecología y Obstetricia no está recomendado cursos repetidos de corticoesteroides. ${ }^{(18)}$

-Antibióticos: No se recomienda su uso de rutina, ya que no prolongan la edad gestacional ni mejoran el pronóstico en mujeres con riesgo de amenaza de parto pretérmino. Deben utilizarse en infecciones que lo ameriten como profilaxis de estreptococo del grupo B intraparto y coriamnionitis. ${ }^{(12)}$

\section{Prevención y recomendaciones}

-Acciones a nivel primario: deben basarse en prevenir y reducir el riesgo en la población ${ }^{(1)}$, así como evitar el consumo de alcohol, drogas y tabaco. Asegurarse una correcta salud bucodental, así como una pesquisa adecuada de infecciones del tracto urinario y bacteriuria asintomática, ha demostrado la prolongación del embarazo de hasta una semana en poblaciones que consumen alimentos ricos en omega 3 , en comparación de aquellas que no la consumen. ${ }^{(8)}$

-Acciones a nivel secundario: se recomienda a las pacientes evitar jornadas laborales muy extensas y con horario nocturno. Además se debe realizar US transvaginal en aquellas mujeres con factores de riesgo y de esta forma diagnosticar quiénes se verían beneficiadas del uso de progesterona.

-Acciones a nivel terciario: realizar el test de fibronectina en pacientes de alto de riesgo de parto pretérmino o aquellas sintomáticas.

\section{CONCLUSIONES}

A pesar de que la prematuridad se considera una de las principales causas de morbimortalidad neonatal, en los últimos años ha disminuido los decesos por esta causa. El principal factor de riesgo para sufrir un parto pretérmino es el antecedente de parto prematuro. Las tres principales causas etiológicas de amenaza de parto pretérmino son de origen idiopático, ruptura de membra- nas y de origen iatrogénico. Los signos y síntomas comunes son poco específicos, por lo cual se prefiere usar la cervicometría y el test de la fibronectina para valorar cuales pacientes se beneficiarán del uso de tocolíticos. No existe tratamiento tocolítico de primera línea, los fármacos que producen menos efectos adversos severos son la indometacina y el atosiban. Se debe de administrar cortircoesteroides a toda mujer con alto riesgo de parto pretérmino, con lo cual se ha demostrado disminución de mortalidad y morbilidad neonatal. Es de suma importancia identificar los factores de riesgo de parto pretérmino en toda gestante, para poder brindar un abordaje adecuado.

\section{BIBLIOGRAFÍA}

1. Gabbe G Niebyl J Leigh J et al. Obstetrics: Normal and Problem Pregnancies. Elsevier Saunders. Philadelphia - USA. $6^{\text {th }}$ edition, 2012

2. Navas L Lankester E. Abordaje del Parto Pretérmino. Rev Méd Costa Rica Centroamérica. 2014; LXXI (613): 725-730.

3. Ochoa A Pérez J. Amenaza de Parto Pretérmino - Rotura Prematura de Membranas - Corioamnioitis. An Sist Sanit Navar. 2009; 32 (1): 105-119.

4. Hospital Clínico Hospital San Joan de Déu. Protocolo: Amenaza de Parto Pretérmino. Universidad de Barcelona. 2015

5. Sociedad Española de Ginecología y Obstetricia. Protocolos Asistenciales en Obstetricia: Amenaza de Parto Pretérmino. proSEGO. 2012

6. Couto D Nápoles D Montes P. Repercusión del Parto Pretermino en la morbilidad y mortalidad perinatales. MEDISAN. 2014; 18 (6): $852-858$

7. Cunningham F Leveno K Bloom S Hauth J Rouse D Spong. Williams Obstetricia. Mc Graw Hill. México. 23ํㅡㄹ Edición, 2011

8. Laterra C Susacasa S Di Marco I Valenti E. Guía de práctica clínica: Amenaza de Parto Pretérmino. Rev Hosp Mat Inf Ramón Sardá. 2011; 31(1): 25-40

9. García A Rosales S Jiménez G. Diagnóstico y Manejo del Parto Pretermino. COMEGO. 2008: 129-149.

10. The American Collegue of Obstetricians and Gynecologist. Trabajo de Parto Prematuro 
y nacimiento prematuro. Pacient Education. 2013

11. Franco P García J Ochoa M Sánchez O Bernardo H. Segura J. Guía de Práctica Clínica: Diagnóstico y Manejo de Parto Pretérmino. Secretaría de Salud de México. 2009

12. Osorio A García J.Guía Farmacoterapeutica de amenaza de parto pretérmino. Rev Colomb Enferm.2015; 10 (10): 78-89

13. Voto L Valenti E Asprea I Voto G Votta R. Parto Pretérmino. FAGO. 2014; 13 (1): 510

14. Ovalle A Kakarieka E Rencoret $\mathrm{G}$ et al. Factores Asociados con el Parto Prematuro entre 22 y 34 semanas en un hospital público de Santiago. Rev Med Chile. 2012; 140: 19-29

15. Robert J Leiva J Germain A. Protocolo de Manejo en pacientes con amenaza de parto pretermino en clínica Las Condes. Rev Med Clin Condes. 2014; 25 (6): 953-957

16. Puerta N. Guía de Practica Clínica de Amenaza de Parto Pretermino. Clinica de la Mujer - Bogotá. 2014.

17. Manzanares S López M Redondo $\mathrm{P}$ et al. Amenaza de Parto Prematuro: valor de la cervicometría y la fibronectina. Actualización Obstétrica y Ginecológica. 2009: 1-8

18. Paris E Sánchez I Beltramino D Copto A. Meneghello Pediatría. Editorial Médica Panamericana. Buenos Aires - Argentina. 6ta edición, 2013

\section{CONFLICTOS DE INTERES}

Los autores declaran que no existe ningún conflicto de interés. 